NOEMI LOURENÇO GIL

\title{
PARTICIPAÇÃO DA CORTICOSTERONA NA INFLAMAÇÃO AGUDA PULMONAR DE RATOS COM BAIXO PESO AO NASCER
}
Dissertação apresentada ao Departamento de Imunologia do Instituto de Ciências Biomédicas da Universidade de São Paulo, para obtenção do Título de Mestre em Ciências.

Área de concentração: Imunologia

Orientador: Prof. Dr. Richardt Gama Landgraf

Versão original 


\section{RESUMO}

GIL, N.L. Participação da corticosterona na inflamação aguda pulmonar de ratos com baixo peso ao nascer. 2016. 82 f. Dissertação (Mestrado em Imunologia) - Instituto de Ciências Biomédicas, Universidade de São Paulo, São Pulo, 2016.

A desnutrição no período gestacional pode comprometer o desenvolvimento fetal e prejudicar a maturação do sistema imune, exercendo efeito negativo prolongado sobre a resposta imunológica. Ratos Wistar de 12 semanas com baixo peso ao nascer, oriundos de mães desnutridas durante a gestação, apresentam resposta inflamatória pulmonar reduzida, e concentração de corticosterona circulante elevada. O presente estudo utilizou-se deste modelo para investigar a influência da corticosterona sobre a resposta inflamatória pulmonar aguda. Retirou-se ambas as adrenais, e após sete dias de reposição de corticosterona em doses fisiológicas, induziu-se lesão pulmonar aguda com LPS i.n. Os resultados obtidos através da contagem total e diferencial de células no lavado broncoalveolar, e da análise histopatológica, mostraram que a redução de corticosterona por adrenalectomia promoveu o aumento do infiltrado de leucócitos para o pulmão em animais de baixo peso ao nascer. Além disso, aumentou-se também a produção de IL-6 e MIP-2 no pulmão, e apesar da produção de TNF- $\alpha$ ser semelhante a dos animais de peso normal ao nascer, a adrenalectomia e reposição de corticosterona em doses fisiológicas promoveu uma maior produção desta citocina nos animais de baixo peso ao nascer; não houve diferença entre os grupos na produção de IL-1 $1 \beta$. Os animais de baixo peso ao nascer desenvolvendo lesão pulmonar aguda passaram a expressar mais COX-2 e 5-LO e a secretar mais $\mathrm{LTB}_{4}$ e $\mathrm{PGE}_{2}$ após a redução de corticosterona por adrenalectomia. Quanto a regulação da corticosterona, notou-se que o ACTH encontrava-se aumentado nos animais que sofreram adrenalectomia, independente do peso ao nascer, e foi reduzido após indução da lesão pulmonar aguda; a expressão pulmonar dos receptores de glicocorticoides apresentou-se reduzida nos animais de baixo peso ao nascer, entretanto, após adrenalectomia e reposição com corticosterona, aumentou-se a expressão dos receptores; não houve diferença entre os grupos na concentração plasmática de transcortina. Por meio destes achados é possível afirmar que as altas concentrações de corticosterona presente em animais de baixo peso ao nascer estão relacionadas a reduzida resposta inflamatória pulmonar aguda.

Palavras-chave: Baixo peso ao nascer. Inflamação. Corticosterona. Desnutrição. Lesão pulmonar aguda. 


\begin{abstract}
GIL, N.L. Influence of corticosterone on acute lung inflammation of low birth weight rats. 2016. 82 p. Masters thesis (Immunology) - Instituto de Ciências Biomédicas, Universidade de São Paulo, São Paulo, 2016.
\end{abstract}

The malnutrition during pregnancy may impair the fetal development and the maturation of immune system, causing prolonged negative effect on immune response. Wistar rats with 12 weeks-old with low birth weight present reduced lung inflammatory response, and increased circulating levels of corticosterone. Using this model, the current work searched investigating the influence of corticosterone upon acute lung inflammatory response. Both adrenals was removed, and after seven consecutive days of corticosterone replacement in physiological levels, the acute lung injury by LPS i.n. was induced. The results obtained by total and differential cells in fluid lavage bronchoalveolar, and histopathological analysis, showed an increase of leucocytes infiltration into lung after adrenalectomy and corticosterone replacement in low birth weight rats. Furthermore, the release of IL-6 and MIP-2 in lung tissue of low birth weight rats with acute lung injury is reduced, however, after adrenalectomy, these cytokines were increased, while TNF- $\alpha$ was similarly released in both groups, however, after adrenalectomy and development of inflammation, this cytokine increased in low birth weight rats; no difference was observed between the groups in IL-1 $\beta$ release. The expression of COX-2 and 5-LO, beyond production of $\mathrm{PGE}_{2}$ and $\mathrm{LTB}_{4}$ in low birth weight rats developing acute lung injury was reduced, nevertheless, after adrenalectomy and corticosterone replacement, these enzymes and lipid mediators were increased. Evaluating the corticosterone regulation, the ACTH was increased in adrenalectomized animals, independing of weight birth, and it was reduced after inflammatory stimulus; the lung expression of glucocorticoid receptors was reduced in low birth weight rats, however, after adrenalectomy and corticosterone replacement the expression of receptors was increased; no difference was observed in plasmatic levels of CBG. Through the data it's possible affirm that high levels of corticosterone are related to reduced acute lung inflammatory response developed by low birth weight rats.

Keywords: Low birth weight. Inflammation. Corticosterone. Subnutrition. Acute lung injury. 


\section{INTRODUÇÃO E REVISÃO DA LITERATURA}

\subsection{Desnutrição e sistema imunológico}

O funcionamento adequado do sistema de defesa é criticamente afetado pela nutrição, de maneira que deficiências nutricionais interferem na resposta mediada por células, função dos fagócitos, produção de citocinas, atividade do Sistema Complemento, e secreção de anticorpos, assim como a afinidade dos mesmos. Alguns estudos sugerem que a desnutrição calórico-proteica (DCP) relaciona-se com a redução da resposta imune regulatória do microambiente de mucosas, potencializando os mecanismos normais de regulação e intolerância imunológica (CUNNINGHAM-RUNDLES; MCNEELEY; MOON, 2005).

Fatores desfavoráveis que comprometem o desenvolvimento fetal podem prejudicar a maturação do sistema imune, exercendo efeito negativo prolongado sobre a resposta imunológica (CHANDRA, 1997; MOORE et al., 2006).

\subsection{Efeitos da desnutrição durante a gestação}

O crescimento normal do feto depende de uma crítica regulação de proliferação celular, organização e diferenciação do embrião, e esse processo depende do perfil genético do embrião, da unidade fetal-placentária-maternal, além de nutrientes e oxigenação que supram adequadamente o desenvolvimento, e a influência hormonal entre mãe e feto (ERGAZ; AVGIL; ORNOY, 2005). BARKER (1990) hipotetizou que o crescimento fetal prejudicado durante o período gestacional está fortemente correlacionado ao desenvolvimento de doenças crônicas na vida tardia.

A restrição do crescimento intrauterino (RCIU) ou a restrição do crescimento fetal (RCF) pode ter diversas etiologias, dentre elas, o uso de drogas de abuso, infecções congênitas, gestação múltipla, hipóxia, deficiências em vitaminas essenciais e minerais, restrição calórica e proteica, e excesso de glicocorticoides (GCs) (ERGAZ et al., 2005; VO; HARDY, 2012). A RCIU/RCF pode resultar num peso ao nascer que é pequeno para a idade gestacional (PIG). 
Estudos epidemiológicos demonstraram que crianças com baixo peso ao nascer (menos que $2500 \mathrm{~g}$ ), apresentam uma incidência aumentada no desenvolvimento de diabetes do tipo II, hipertensão e doenças cardiovasculares (HALES et al., 1991; HUXLEY et al., 2007; JAQUET et al., 2000). Ainda, modelos animais de RCIU reforçam a hipótese de Barker, pois mediante o desenvolvimento in utero prejudicado, apresentaram comprometimento no metabolismo da glicose, colesterol e triglicérides na vida adulta (DAHRI et al., 1991; LANGLEY; BROWNE; JACKSON, 1994; LUCAS et al., 1996; SOHI et al., 2011).

Os PIGs apresentam uma resposta imunológica reduzida além de imunocompetência prejudicada que pode permanecer durante anos. Infecções no trato respiratório inferior e superior são três vezes mais frequentes em crianças PIGs (CHANDRA, 2002).

Neel (1962) descreveu a hipótese do fenótipo poupador, enquanto Barker (1991) a estabeleceu: a restrição alimentar materna, durante a gestação, provoca mudanças no armazenamento de energia pelo feto, adaptando-o ao ambiente intrauterino. $O$ feto responde com retardo no crescimento quando faltam nutrientes em seu ambiente. Essa adaptação permanece na vida pós-natal, de maneira que, ao se deparar com uma oferta maior de nutrientes, é gerado um desequilíbrio, que pode acarretar no desenvolvimento de doenças cardiometabólicas. Além disso, estas alterações permaneceriam e seriam transmitidas a gerações futuras.

Durante o cerco alemão na Holanda, entre o fim de 1944 e início de 1945, na segunda guerra mundial, uma severa restrição alimentar foi provocada em mulheres gestantes. Os fetos que sofreram desnutrição no primeiro trimestre de gestação desenvolveram obesidade na vida adulta, enquanto os que sofreram restrição em estágios posteriores do período gestacional, tiveram diversas outras consequências, como por exemplo, maior predisposição à doenças cardiovasculares (KYLE; PICHARD, 2006).

Surgiu posteriormente a hipótese da resposta adaptativa-preditiva, sugerindo que, o organismo em fase de desenvolvimento, placentário ou em lactação, através de sinais hormonais maternos, prevê o ambiente no qual crescerá, fazendo com que sua fisiologia seja ajustada para tal. Na maturidade, ao se deparar com o ambiente não previsto, há o aumento no risco de desenvolvimento de doenças que provavelmente irão se manifestar após o período reprodutivo (GLUCKMAN; HANSON, 2004; GLUCKMAN; HANSON; BEEDLE, 2007). Há também a 
hipótese de que a desnutrição materna e a insuficiência placentária resultam em desnutrição fetal, com consequente diminuição do crescimento do feto (JANSSON, T.; POWELL, 2007). Sabe-se que a desnutrição materna aumenta os níveis de GCs

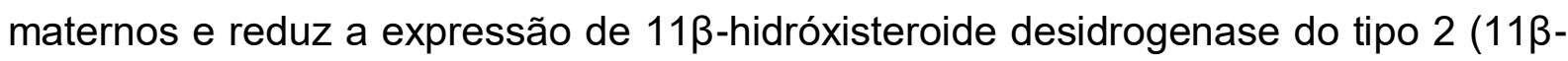
HSD2) placentário, aumentando a exposição do feto aos GCs maternos, causando alterações que podem se perpetuar durante gerações (EDWARDS et al., 1993; SECKL, 2004).

Diferentes modelos animais com abordagem de intervenção nutricional tem sido de grande valia para se compreender os fatores e as consequências da RCF, dentre eles, ratos e camundongos compartilham características genéticas, bioquímicas, estruturais e fisiológicas com humanos, o que auxilia na compreensão dos desafios nutricionais enfrentados em países subdesenvolvidos (VUGUIN, 2007).

\subsection{Inflamação}

O processo inflamatório é desencadeado frente a necessidade de sobrevivência do tecido perante dano ou lesão causados por algum agente infeccioso ou não (RYAN; MAJNO, 1977). Há indutores exógenos microbianos da inflamação, como patógenos e fatores de virulência, assim como não microbianos, que podem ser: consumo de álcool, tabaco e dieta hipercalórica. Fatores endógenos oriundos de tecido danificado, sob estresse, ou em mal funcionamento, também podem desencadear uma resposta inflamatória, porém estes sinais ainda carecem de informações (MEDZHITOV, 2008).

A resposta inflamatória caracteriza-se por mudanças na homeostase tecidual e fluxo sanguíneo, por secreção e ação de mediadores inflamatórios mediante ativação e migração de células do sistema imunológico (HOUCK, 1976). As manifestações clínicas típicas descritas são: calor, dor, rubor e edema, decorrentes da ação de mediadores na microcirculação da região (LAWRENCE; WILLOUGHBY; GILROY, 2002; RATHER, 1971). A vasodilatação que ocorre nas arteríolas permite maior aporte sanguíneo para a região inflamada provocando calor e rubor. Há aumento de permeabilidade e da expressão das moléculas de adesão nas vênulas para permitir o extravasamento do plasma dos vasos para o tecido, e, a migração de células para o foco inflamatório. Este líquido acumulado nos tecidos é que ocasiona 
o edema, podendo pressionar as terminações nervosas causando dor, que também pode ser resultado da ação de mediadores inflamatórios (LAWRENCE et al., 2002).

Inflamações agudas são geralmente autolimitantes, se instalando apenas com o intuito de restabelecer o equilíbrio fisiológico. É o que ocorre em casos de infecção e lesão tecidual em que o agente é removido, e o tecido, reparado. Entretanto, há situações em que o processo inflamatório pode se prolongar, passando a ser crônico e prejudicial ao organismo (NATHAN; DING, 2010). Os mecanismos de inflamação induzidos por infecção são melhores compreendidos do que os processos inflamatórios desencadeados por outros indutores. Os mecanismos de estados inflamatórios crônicos sistêmicos, como por exemplo, doenças autoimunes, síndromes metabólicas e neoplasias, ainda necessitam de mais estudos, e portanto, não cabem neste padrão clássico de transição entre inflamação aguda e crônica (ALESSANDRI et al., 2013; FREIRE; VAN DYKE, 2013; MEDZHITOV, 2008).

\subsubsection{Células envolvidas na inflamação aguda - imunidade inata}

Algumas células originadas na medula óssea, de precursores mieloides, podem se diferenciar para linhagem granulocítica, dando origem aos neutrófilos. Após maturação, parte dos neutrófilos permanecem na medula, enquanto outra vai para a circulação, onde sobrevivem durante algumas horas. São as primeiras células a migrarem para o foco inflamatório em grande número (DE KLEER et al., 2014; OHLS et al., 1995).

Os precursores de origem mieloide que se diferenciam em pró-monócitos vão para o sangue, onde são chamados de monócitos. Alguns migram para diferentes tecidos onde se diferenciam para macrófagos e residem por longo período de tempo (DE KLEER et al., 2014; SCHULZ et al., 2012; VAN FURTH, 1980).

A resposta inflamatória pode ser iniciada quando os macrófagos residentes em contato com algum patógeno são ativados liberando citocinas e quimiocinas que agem sobre a microcirculação, atraindo e permitindo a passagem de neutrófilos através dos vasos (FREIRE; VAN DYKE, 2013; LEY et al., 2007). Os mastócitos, células do tecido conjuntivo, presentes nos vasos sanguíneos, também podem participar quando ativados, pois nessa condição, liberam substâncias vasoativas, dentre elas, mediadores lipídicos (FREIRE; VAN DYKE, 2013). 
Estes leucócitos apresentam receptores de reconhecimento de padrões (PRRs), que por sua vez, reconhecem padrões moleculares associados a patógenos (PAMPS) ou padrões moleculares associados ao dano (DAMPS), sendo que o último pode ativar as mesmas proteínas que o primeiro (MILLS, 2011).

\subsubsection{Receptores do tipo Toll (TLRs)}

Os TLRs são proteínas transmembrânicas de única passagem, com domínios externos repetidos ricos em leucina que medeiam o reconhecimento dos PAMPS e DAMPS, e domínios intracelulares de receptores de Toll de interleucina 1(IL-1), os chamados TIR, os quais interagem com outros domínios do tipo TIR presentes em outras moléculas. Estes receptores estão presentes na membrana plasmática, endossomal, lisossomal e endolisossomal (AKIRA; UEMATSU; TAKEUCHI, 2006; KOVACH; STANDIFORD, 2011).

Amplamente conservados entre as espécies, dentre 10 TLRs funcionais encontrados em humanos, e 12 em camundongos, estudos mostraram que há uma molécula em cada espécie em comum que apresenta alto grau de similaridade entre si. Os TLRs são capazes de reconhecer lipídios, lipoproteínas, proteínas e ácidos nucleicos presentes em diversos patógenos tais como bactérias, vírus e fungos (AKIRA et al., 2006).

A ativação de TLRs desencadeia diferentes tipos de vias de sinalização, culminando na ação de distintos fatores de transcrições, resultando na produção de citocinas, fatores quimiotáticos e peptídeos antimicrobianos, por exemplo (AKIRA et al., 2006; KAWAl; AKIRA, 2010; KOVACH; STANDIFORD, 2011).

O receptor TLR4 foi o primeiro membro da família de TLRs a ser descrito. Este reconhece o lipopolissacarídeo (LPS) presente na parede celular de células bacterianas gram-negativas, as quais podem levar ao choque séptico (KAWAl; AKIRA, 2010). 


\subsubsection{Resposta imunológica ao LPS}

O Lipopolissacarídeo é o maior componente externo à membrana de bactérias gram-negativas, e apresenta a função de conferir resistência a perigos externos, sendo de extrema importância para a sobrevivência destes microrganismos. Uma E.coli contém cerca de 3,5 $\times 10^{6}$ moléculas de LPS (LEONE et al., 2007; RIETSCHEL et al., 1994).

Quando ocorre lise ou crescimento bacteriano, o LPS é liberado, deixando o lipídio $A$ exposto, por meio do qual se inicia a resposta imunológica (VAN AMERSFOORT; VAN BERKEL; KUIPER, 2003). O LPS livre é então capaz de ativar TLR4 desencadeando um processo de sinalização intracelular, com consequente ativação do fator de transcrição nuclear fator $\mathrm{kB}(\mathrm{NF}-\mathrm{kB})$, que por sua vez, leva a produção de citocinas pró-inflamatórias como a IL-6 e TNF- $\alpha$ (KAGAN et al., 2008; TANIMURA et al., 2008).

\subsubsection{Mediadores inflamatórios}

Os mediadores envolvidos com o processo inflamatório podem ser oriundos das proteínas do plasma, ou secretados pelas células locais. A concentração plasmática dos mediadores pode aumentar acentuadamente como resultado da secreção elevada de precursores pelos hepatócitos durante a resposta de fase aguda. De acordo com suas propriedades bioquímicas podem ser classificados em sete grupos: citocinas, quimiocinas, aminas vasoativas, peptídeos vasoativos, fragmentos de componentes do sistema complemento, enzimas proteolíticas e mediadores lipídicos (FREIRE; VAN DYKE, 2013; MEDZHITOV, 2008;2010).

Citocinas pró-inflamatórias são secretadas por diferentes tipos de células e são pleiotrópicas, exercendo atividades como: ativação de leucócitos e endotélio. Exemplo de citocinas envolvidas com a resposta de fase aguda são: fator de necrose tumoral- $\alpha$ (TNF- $\alpha$ ), interleucina-1 (IL-1) e interleucina-6 (IL-6), sendo secretadas nesta fase principalmente por macrófagos e mastócitos. As quimiocinas, também secretadas por diversos tipos celulares agem como substâncias atrativas de leucócitos para o sítio inflamado (STRIZ et al., 2014). 
$\mathrm{O}$ aumento intracelular de íons $\mathrm{Ca}^{2+}$ pode promover a ação da enzima fosfolipase $A_{2}\left(F L A_{2}\right)$ sobre os fosfolipídeos de membrana, gerando o ácido araquidônico, que é metabolizado para a forma de eicosanóides pela ação das enzimas cicloxigenase 1 ou 2 (COX-1 ou COX-2) gerando as prostaglandinas e tromboxanos, ou pode ser metabolizado pela 5-lipoxigenase (5-LO), gerando os leucotrienos e lipoxinas, como ilustrado pela figura 1 (MEDZHITOV, 2008).

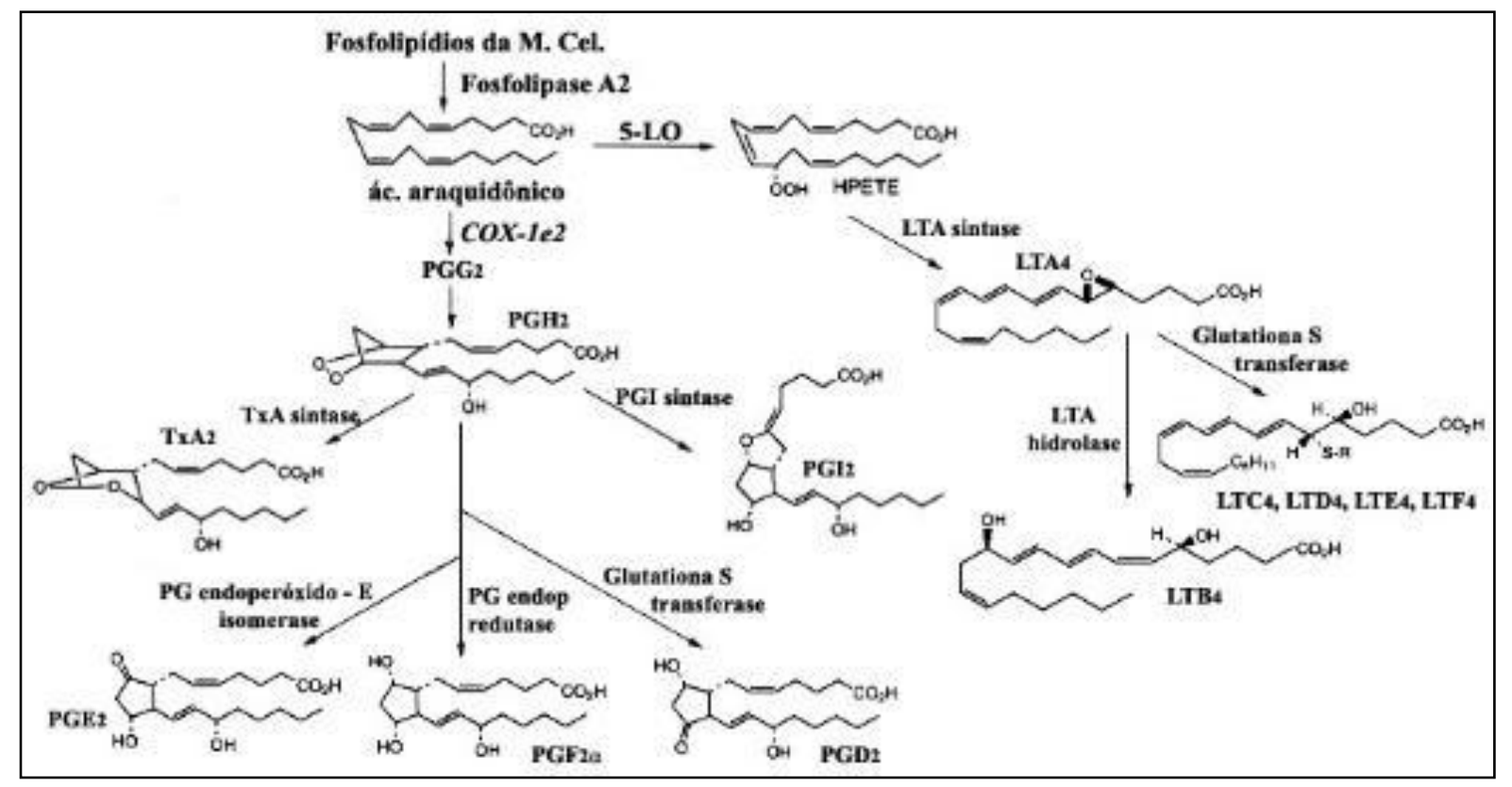

Figura 1 - Representação da cascata do ácido araquidônico. A FLA 2 age sobre os fosfolipídeos de membrana resultando na formação do ácido araquidônico. Este, por sua vez, mediante ação das enzimas COX-1 ou 2, gera as prostaglandinas e tromboxanos, tendo formação de lipídios intermediários e ação de outras enzimas antes de chegar ao produto final. Quando o ác. araquidônico é catalisado pela 5-LO são gerados os leucotrienos, tendo como produto intermediário o LTA 4 . Adaptação de Kuehl, Egan (1980) .

As prostaglandinas agem sinergicamente com outros mediadores contribuindo para a ocorrência das manifestações clínicas da inflamação, como o edema, por serem vasodilatadores potentes, apesar de não aumentarem a permeabilidade vascular, e dor, por diminuir o limiar de receptores algésicos, causando hiperalgesia (PERRETTI; AHLUWALIA, 2000; SUGIMOTO; NARUMIYA, 2007; WILLIAMS; MORLEY, 1973). São também potentes agentes piréticos. A prostaglandina $E_{2}$ $\left(\mathrm{PGE}_{2}\right)$ é capaz de causar febre quando produzida por células não neuronais, provavelmente as endoteliais dos vasos que perfundem o hipotálamo, em resposta 
ao estímulo de IL-1 produzida por macrófagos perante infecções bacterianas e virais (NAKAMURA, 2011; SAPER; ROMANOVSKY; SCAMMELL, 2012).

Dentre os produtos da ação da 5-LO, o Leucotrieno $\mathrm{B}_{4}\left(\mathrm{LTB}_{4}\right)$ exerce função importante sobre os neutrófilos como agente quimiotático e agente aderente ao endotélio. Além disso, o $\mathrm{LTB}_{4}$ tem grande importância na imunidade inata por aumentar a atividade microbicida de macrófagos ativando a Nicotinamida adenina dinucleotídeo fosfato, forma oxidada (NADPH-oxidase), e portanto, a produção de espécies reativas de oxigênio (LE BEL; BRUNET; GOSSELIN, 2014).

\subsubsection{Migração de neutrófilos para o foco inflamatório}

Como mencionado, nas vênulas pós-capilares localizadas na região do foco inflamatório é que ocorre a migração transendotelial dos leucócitos através das interações entre as moléculas de adesão. As primeiras células a migrarem são os neutrófilos, e a primeira etapa envolvida é a interação entre a L-selectina presente na membrana destas células, com moléculas expressas pelas células endoteliais, de maneira que os neutrófilos diminuem a velocidade de rolamento até estabelecerem contato mais estável com o endotélio (WILKINSON, 1974; WILKINSON; MCKAY, 1974).

Em segunda instância, quimiocinas associadas à membrana das células endoteliais ativam os neutrófilos, fazendo com que a integrina expressa pelo neutrófilo, antígeno 1 associado à função leucocitária (LFA-1), adquira conformação de alta afinidade pela molécula de adesão intercelular 1 (ICAM-1) presente no endotélio, e isso possibilita que os neutrófilos possam aderir e se espraiar sobre o endotélio (APLIN et al., 1998; GONZALEZ-AMARO; SANCHEZ-MADRID, 1999).

Após a adesão, os neutrófilos então passam pelas junções entre as células do endotélio por meio da interação entre as moléculas de adesão celular endotelial plaquetária (PECAM) presentes em ambos os tipos celulares. Fora dos vasos, os neutrófilos continuam migrando para o local de inflamação por quimiotaxia (PETRI; PHILLIPSON; KUBES, 2008).

A migração transendotelial de neutrófilos depende da ação de mediadores inflamatórios, como por exemplo, o $\mathrm{LTB}_{4}$, quimiocinas como IL-8 e proteína 
inflamatória de macrófagos 2 (MIP-2), e as citocinas TNF- $\alpha$ e IL-1 (BAGGIOLINI, 1998; BAGGIOLINI; LOETSCHER, 2000).

\subsection{Hormônios glicocorticoides}

\subsubsection{Adrenais}

As glândulas adrenais localizam-se anatomicamente sobre cada um dos rins, envolvidas por tecido adiposo, e são compostas por duas camadas: o córtex adrenal (parte externa) e a medula adrenal (parte interna). O córtex adrenal é composto por três zonas (glomerulosa, fasciculada e reticular) que produzem diversos hormônios esteroides derivados do colesterol: mineralocorticoides, glicocorticoides e hormônios sexuais (KEMPNA; FLUCK, 2008).

O principal mineralocorticoide é a aldosterona, que age nos túbulos distais e coletores dos rins, promovendo a retenção de $\mathrm{Na}^{+}$e eliminação de $\mathrm{K}^{+}$. Sendo assim, a ausência deste hormônio provoca perda de $\mathrm{Na}^{+}$, que é retentor de água, e consequente choque circulatório, podendo levar o indivíduo a morte (SIMPSON; TAIT; BUSH, 1952). Sua secreção é aumentada pela atividade do sistema ReninaAngiotensina quando há redução de $\mathrm{Na}^{+}$na circulação e queda da pressão arterial (MULROW; FRANCO-SAENZ, 1996).

Apesar da produção de hormônios sexuais ocorrerem majoritariamente nas gônadas, o córtex da adrenal também produz estes hormônios, tanto do sexo correspondente, como do sexo oposto. A desidroepiandrosterona (DHEA) possui maior importância biológica, com menor androgeneidade em comparação à testosterona no sexo masculino, porém, com significância fisiológica androgênica no sexo feminino (CASSON et al., 2000; ORENTREICH et al., 1984).

A medula adrenal é composta por células cromafins, as quais consistem em neurônios simpáticos pós-ganglionares modificados, pois liberam seus neurotransmissores, neste caso, hormônios, diretamente na circulação. As catecolaminas (epinefrina e norepinefrina), armazenadas nos grânulos cromafins, são liberadas após impulso simpático sobre a adrenal em condições de medo ou estresse (GOLDSTEIN; EISENHOFER; KOPIN, 2003; TANK; LEE WONG, 2015). 


\subsubsection{Glicocorticoides}

Os glicocorticoides (GCs) foram assim nomeados por conta do seu efeito sobre o metabolismo dos carboidratos, porém, regulam diversas outras funções, dentre elas, desenvolvimento e inflamação. Estes hormônios são sintetizados e liberados pelo córtex adrenal, num ritmo circadiano, em resposta a algum estímulo fisiológico e ao estresse (MILLER; AUCHUS, 2011; RHEN; CIDLOWSKI, 2005; SAPOLSKY, 1996).

O eixo hipotálamo-pituitária-adrenal (HPA) regula a concentração dos GCs na circulação, como ilustrado pela figura 2. Sob estímulo, o núcleo paraventricular do hipotálamo libera o hormônio liberador de corticotropina $(\mathrm{CRH})$ que age na pituitária anterior, estimulando a liberação do hormônio adrenocorticotrófico (ACTH). Os GCs são então rapidamente sintetizados e liberados quando o ACTH estimula o córtex adrenal (WEBSTER et al, 2002). O sistema HPA é balanceado pelo efeito de feedback negativo que os GCs exercem ao agir sobre a pituitária anterior e hipotálamo, sinalizando para a diminuição da secreção de $\mathrm{CRH}$, e, consequentemente, de ACTH (JOHN; BUCKINGHAM, 2003; MALKOSKI; DORIN, 1999). 


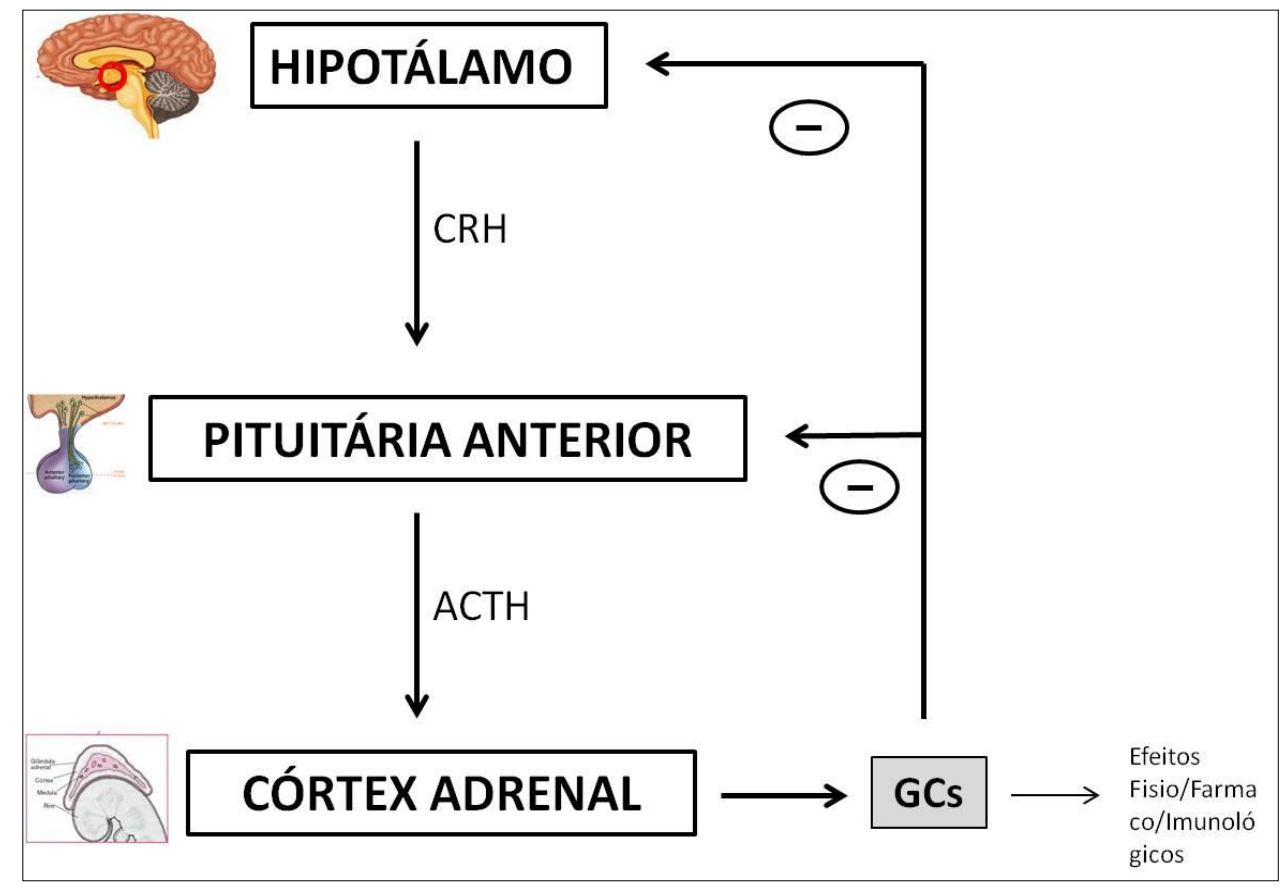

Figura 2 - llustração do sistema Hipotálamo-pituitária-adrenal. O $\mathrm{CRH}$ é secretado pelo Hipotálamo perante algum estímulo, e age na pituitária anterior estimulando-a a secretar o ACTH, que por sua vez, age sobre o córtex adrenal fazendo com que este sintetize rapidamente os GCs para serem secretados. O mecanismo normal de regulação consiste em um feedback negativo do GC, que em alta concentração sinaliza para o Hipotálamo e Pituitária reduzirem a secreção de CRH e ACTH respectivamente. Adaptação de De Guia, Rose e Herzig (2014).

O eixo HPA é o sistema central de resposta ao estresse, de maneira que sua regulação prejudicada foi relacionada a diversas patologias, tais quais 0 desenvolvimento de autoimunidades, hipertensão, desordens afetivas e, principalmente, depressão (BREUNER; ORCHINIK, 2002).

Os principais GCs ativos são o cortisol em humanos, e a corticosterona (CORT) em murinos. Por serem substâncias lipídicas, com baixa solubilidade no plasma, são transportados por uma proteína glicosilada, denominada globulina ligadora de corticosteroide (CBG), denominada também de transcortina, que contém um único sítio de ligação para GCs e progesterona, ambos com alta afinidade (GARREL, 1996; PUGEAT et al., 1989). Somente os GCs circulantes livres, cerca de $5 \%$, é que são capazes de exercer efeito fisiológico, sendo que de $80-90 \%$ estão ligados à CBG, e o remanescente, à albumina (SIITERI et al., 1982). Secretada primeiramente pelos hepatócitos, a CBG é uma proteína de fase aguda negativa, pois durante o processo inflamatório, inclusive na sepse, sua concentração 
plasmática é diminuída, provavelmente para se aumentar a quantidade de GCs livres (PUGEAT et al., 1989).

Os GCs exercem suas funções fisiológicas e farmacológicas através do receptor de glicocorticoide $(\mathrm{GR})$, que pertence à família de receptores nucleares. Trata-se de uma proteína modular, constituída por três domínios funcionais: um central ligante de DNA (DBD), domínio N-terminal de transativação (NTD), e o domínio de ligação ao ligante C-terminal (LDB) (KUMAR; THOMPSON, 2005).

Os GRs, quando na ausência dos ligantes, se situam no citoplasma, associados a um complexo multiproteico, que contém proteínas chaperonas e imunofilinas (GRAD; PICARD, 2007). A ligação do hormônio ao seu receptor faz com que este se dissocie do complexo e se transloque para o núcleo da célula, podendo agir diretamente como um fator de transcrição gênica, ou transreprimindo a atividade transcricional de outros fatores, como a proteína ativadora 1 (AP-1) e NF-kB, envolvidos com a transcrição de genes relacionados ao processo inflamatório (ROGATSKY; IVASHKIV, 2006).

\subsubsection{Efeitos Fisiológicos, Farmacológicos e Imunológicos da ação dos GCs}

Os GCs provocam aumento da concentração de glicose no sangue utilizando estoques de proteína e gordura: estimula a gliconeogênese hepática; inibe a absorção de glicose por muitos tecidos, exceto o cérebro; estimula a degradação de proteínas principalmente nos músculos e facilita a lipólise (RHEN; CIDLOWSKI, 2005).

Estes hormônios apresentam ampla ação imunomodulatória, sendo uma das drogas mais prescritas mundialmente (SCHACKE; DOCKE; ASADULLAH, 2002). Os GCs agem aumentando a transcrição de genes anti-inflamatórios e diminuindo a de genes inflamatórios (BARNES, 1998). O Resultado desta ação pode ser a inibição da produção de mediadores lipídicos e citocinas, tais como a IL-1ß, IL-2, IL-6, TNFa, GM-CSF, e de quimiocinas, como a IL-8 (FLOWER; ROTHWELL, 1994; NELSON et al., 1993; NORTHROP; ULLMAN; CRABTREE, 1993). Além disso, os GCs podem interferir na expressão das moléculas de adesão, por exemplo, a ICAM-1 e E-selectina, indiretamente, pela redução da liberação de citocinas, e diretamente, reprimindo a transcrição gênica (CRONSTEIN et al., 1992). 


\subsection{Justificativa}

Doenças cardiometabólicas são as mais discutidas em se tratando de adversidades intrauterinas, entretanto, outras enfermidades têm sido estudadas, por exemplo, a asma (FISHER; STEELE; KARROW, 2012; LANDGRAF et al., 2008). Pouco ainda se sabe sobre os mecanismos envolvidos nas alterações do sistema imunológico de um indivíduo que sofreu restrição alimentar in utero.

Alguns dados obtidos anteriormente mostram que ratos Wistar desnutridos intrauterinamente apresentam menor número de leucócitos circulantes, além de reduzida expressão das moléculas de adesão P-selectina e ICAM-1 perante estímulo, e reduzida expressão basal de L-selectina nos leucócitos. Foi visto ainda, que ratos Wistar adultos (12 semanas), oriundos de mães desnutridas durante a gestação, apresentam atenuamento da resposta inflamatória alérgica, caracterizada pelo menor infiltrado de eosinófilos no lavado broncoalveolar (BAL). Hipotetizou-se que esta reduzida resposta inflamatória poderia estar relacionada aos altos níveis circulantes de CORT que estes animais apresentam (LANDGRAF et al., 2005; LANDGRAF et al. 2008; LANDGRAF et al. 2012). 


\section{CONCLUSÃO}

Os dados obtidos no presente estudo permitem concluir que a concentração elevada de CORT, presente em animais de BPN, está modulando negativamente a resposta inflamatória pulmonar aguda. Ainda que a redução de CORT nos animais com BPN desenvolvendo LPA resultou em aumento dos mediadores inflamatórios que estavam com sua produção diminuída, alguns efeitos observados não estão totalmente compreendidos. É possível então supor que, além da concentração elevada de CORT, os animais de BPN podem apresentar outras alterações que interferem na resposta imunológica aguda, como atividade dos fagócitos e regulação do sistema nervoso autônomo simpático, entretanto, outros estudos se fazem necessários para confirmação. 


\section{REFERÊNCIAS*}

AEFFNER, F.; BOLON, B.; DAVIS, I. C. Mouse Models of Acute Respiratory Distress Syndrome: A Review of Analytical Approaches, Pathologic Features, and Common Measurements. Toxicol. Pathol., v. 43, n. 8, p. 1074-1092, Dec 2015.

AKIRA, S.; UEMATSU, S.; TAKEUCHI, O. Pathogen recognition and innate immunity. Cell, v. 124, n. 4, p. 783-801, Feb 2006.

ALESSANDRI, A. L. et al. Resolution of inflammation: mechanisms and opportunity for drug development. Pharmacol. Ther., v. 139, n. 2, p. 189-212, Aug 2013.

ALEXANDER, B. N.; FEWELL, J. Metyrapone restores the febrile response to Escherichia coli LPS in pregnant rats. Am. J. Physiol. Regul. Integr. Physiol., v. 300, n. 6, p. 1588-1595, Jun 2011.

ANTONELLI, M. et al. Leukotrienes and alpha tumor necrosis factor levels in the bronchoalveolar lavage fluid of patient at risk for the adult respiratory distress syndrome. Minerva Anestesiol., v. 60, n. 9, p. 419-426, Sep 1994.

APLIN, A. E. et al. Signal transduction and signal modulation by cell adhesion receptors: the role of integrins, cadherins, immunoglobulin-cell adhesion molecules, and selectins. Pharmacol. Rev., v. 50, n. 2, p. 197-263, Jun 1998.

BAGGIOLINI, M. Chemokines and leukocyte traffic. Nature, v. 392, n. 6676, p. 565568, Apr 1998.

BAGGIOLINI, M.; LOETSCHER, P. Chemokines in inflammation and immunity. Immunol. Today, v. 21, n. 9, p. 418-420, Sep 2000.

BAILEY, J. M. New mechanisms for effects of anti-inflammatory glucocorticoids. Biofactors, v. 3, n. 2, p. 197-102, Jun 1991.

BARKER, D. J. The fetal and infant origins of adult disease. BMJ, v. 301, n. 6761, p. 1111, Nov 171990.

\footnotetext{
* De acordo com: ASSOCIAÇÃO BRASILEIRA DE NORMAS TÉCNICAS. NBR 6023: informação e documentação: referências: elaboração. Rio de janeiro, 2002.
} 
BARKER, D. J. The intrauterine origins of cardiovascular and obstructive lung disease in adult life. The Marc Daniels Lecture 1990. J. R. Coll. Physicians. Lond., v. 25, n. 2, p. 129-133, Apr 1991.

BARNES, P. J. Anti-inflammatory actions of glucocorticoids: molecular mechanisms. Clin. Sci. (Lond.), v. 94, n. 6, p. 557-572, Jun 1998.

BECKER, J. L.; GRASSO, R. J.; DAVIS, J. S. Dexamethasone action inhibits the release of arachidonic acid from phosphatidylcholine during the suppression of yeast phagocytosis in macrophage cultures. Biochem. Biophys. Res. Commun., v. 153, n. 2, p. 583-590, Jun 1988.

BELKACEMI, L. et al. Altered placental development in undernourished rats: role of maternal glucocorticoids. Reprod. Biol. Endocrinol., v. 9, p. 105, Aug 2011.

BERTINI, R.; BIANCHI, M.; GHEZZI, P. Adrenalectomy sensitizes mice to the lethal effects of interleukin 1 and tumor necrosis factor. J. Exp. Med., v. 167, n. 5, p. 17081712, May 1988.

BOERSMA, B.; WIT, J. M. Catch-up growth. Endocr. Rev., v. 18, n. 5, p. 646-661, Oct 1997.

BRAWLEY, L. et al. Glycine rectifies vascular dysfunction induced by dietary protein imbalance during pregnancy. J. Physiol., v. 554, n. Pt 2, p. 497-504, Jan 2004.

BREUNER, C. W.; ORCHINIK, M. Plasma binding proteins as mediators of corticosteroid action in vertebrates. J. Endocrinol., v. 175, n. 1, p. 99-112, Oct 2002.

BURGER, D.; DAYER, J. M. Inhibitory cytokines and cytokine inhibitors. Neurology, v. 45, n. 6 Suppl 6, p. S39-43, Jun 1995.

CAMPOLONGO, P. et al. Endocannabinoids in the rat basolateral amygdala enhance memory consolidation and enable glucocorticoid modulation of memory. Proc. Natl. Acad. Sci. U. S. A., v. 106, n. 12, p. 4888-4893, Mar 2009.

CASSON, P. R. et al. Dehydroepiandrosterone supplementation augments ovarian stimulation in poor responders: a case series. Hum. Reprod., v. 15, n. 10, p. 21292132, Oct 2000.

CAVALCANTI, D. M. et al. Adrenal deficiency alters mechanisms of neutrophil mobilization. Mol. Cell. Endocrinol., v. 249, n. 1-2, p. 32-39, Apr 2006. 
CHANDRA, R. K. Nutrition and the immune system: an introduction. Am. J. Clin. Nutr., v. 66, n. 2, p. 460S-463S, Aug 1997.

CHANDRA, R. K. Nutrition and the immune system from birth to old age. Eur. J. Clin. Nutr., v. 56 Suppl 3, p. S73-76, Aug 2002.

CHUANG, K. H. et al. Attenuation of LPS-induced lung inflammation by glucosamine in rats. Am. J. Respir. Cell. Mol. Biol., v. 49, n. 6, p. 1110-1119, Dec 2013.

CORREIA-BRANCO, A.; KEATING, E.; MARTEL, F. Maternal undernutrition and fetal developmental programming of obesity: the glucocorticoid connection. Reprod. Sci., v. 22, n. 2, p. 138-145, Feb 2015.

CRONSTEIN, B. N. et al. A mechanism for the antiinflammatory effects of corticosteroids: the glucocorticoid receptor regulates leukocyte adhesion to endothelial cells and expression of endothelial-leukocyte adhesion molecule 1 and intercellular adhesion molecule 1. Proc. Natl. Acad. Sci. U. S. A., v. 89, n. 21, p. 9991-9995, Nov 1992.

CUNNINGHAM-RUNDLES, S.; MCNEELEY, D. F.; MOON, A. Mechanisms of nutrient modulation of the immune response. J. Allergy Clin. Immunol., v. 115, n. 6, p. 1119-1128; quiz 1129, Jun 2005.

DA, J.; CHEN, L.; HEDENSTIERNA, G. Nitric oxide up-regulates the glucocorticoid receptor and blunts the inflammatory reaction in porcine endotoxin sepsis. Crit. Care Med., v. 35, n. 1, p. 26-32, Jan 2007.

DAHRI, S. et al. Islet function in offspring of mothers on low-protein diet during gestation. Diabetes, v. 40 Suppl 2, p. 115-120, Dec 1991.

DE GUIA, R. M.; ROSE, A. J.; HERZIG, S. Glucocorticoid hormones and energy homeostasis. Horm. Mol. Biol. Clin. Investig., v. 19, n. 2, p. 117-128, Aug 2014.

DE KLEER, I. et al. Ontogeny of myeloid cells. Front. Immunol., v. 5, p. 423, 2014.

EDWARDS, C. R. et al. Dysfunction of placental glucocorticoid barrier: link between fetal environment and adult hypertension? Lancet, v. 341, n. 8841, p. 355-357, Feb 1993.

ERGAZ, Z.; AVGIL, M.; ORNOY, A. Intrauterine growth restriction-etiology and consequences: what do we know about the human situation and experimental animal models? Reprod. Toxicol., v. 20, n. 3, p. 301-322, Sep-Oct 2005. 
EVANS, G. F.; ZUCKERMAN, S. H. Glucocorticoid-dependent and -independent mechanisms involved in lipopolysaccharide tolerance. Eur. J. Immunol., v. 21, n. 9, p. 1973-1979, Sep 1991.

FARSKY, S. P.; SANNOMIYA, P.; GARCIA-LEME, J. Secreted glucocorticoids regulate leukocyte-endothelial interactions in inflammation. A direct vital microscopic study. J. Leukoc. Biol., v. 57, n. 3, p. 379-386, Mar 1995.

FISHER, R. E.; STEELE, M.; KARROW, N. A. Fetal programming of the neuroendocrine-immune system and metabolic disease. J. Pregnancy, v. 2012, p. 792934, 2012.

FLOWER, R. J.; ROTHWELL, N. J. Lipocortin-1: cellular mechanisms and clinical relevance. Trends Pharmacol. Sci., v. 15, n. 3, p. 71-76, Mar 1994.

FRANCO, Mdo. C. et al. Intrauterine undernutrition: expression and activity of the endothelial nitric oxide synthase in male and female adult offspring. Cardiovasc. Res., v. 56, n. 1, p. 145-163, Oct 2002.

FREIRE, M. O.; VAN DYKE, T. E. Natural resolution of inflammation. Periodontol. 2000, v. 63, n. 1, p. 149-164, Oct 2013.

GARREL, D. R. Corticosteroid-binding globulin during inflammation and burn injury: nutritional modulation and clinical implications. Horm. Res., v. 45, n. 3-5, p. 245-251, 1996.

GLUCKMAN, P. D.; HANSON, M. A. Maternal constraint of fetal growth and its consequences. Semin. Fetal Neonatal Med., v. 9, n. 5, p. 419-425, Oct 2004.

GLUCKMAN, P. D.; HANSON, M. A.; BEEDLE, A. S. Early life events and their consequences for later disease: a life history and evolutionary perspective. Am. J. Hum. Biol., v. 19, n. 1, p. 1-19, Jan-Feb 2007.

GOLDSTEIN, D. S.; EISENHOFER, G.; KOPIN, I. J. Sources and significance of plasma levels of catechols and their metabolites in humans. J. Pharmacol. Exp. Ther., v. 305, n. 3, p. 800-811, Jun 2003.

GONZALEZ-AMARO, R.; SANCHEZ-MADRID, F. Cell adhesion molecules: selectins and integrins. Crit. Rev. Immunol., v. 19, n. 5-6, p. 389-429, 1999. 
GRAD, I.; PICARD, D. The glucocorticoid responses are shaped by molecular chaperones. Mol. Cell. Endocrinol., v. 275, n. 1-2, p. 2-12, Sep 2007.

GUO, Z. et al. Prevention of LPS-induced acute lung injury in mice by progranulin. Mediators Inflamm., v. 2012, p. 540794, 2012.

HALES, C. N. et al. Fetal and infant growth and impaired glucose tolerance at age 64. BMJ, v. 303, n. 6809, p. 1019-1022, Oct 1991.

HOFMAN, P. L. et al. Premature birth and later insulin resistance. N. Engl. J. Med., v. 351, n. 21, p. 2179-2186, Nov 2004.

HOUCK, J. C. Inflammation: a quarter century of progress. J. Invest. Dermatol., v. 67, n. 1, p. 124-128, Jul 1976.

HUDSON, L. D. et al. Clinical risks for development of the acute respiratory distress syndrome. Am. J. Respir. Crit. Care Med., v. 151, n. 2 Pt 1, p. 293-301, Feb 1995.

HUXLEY, R. et al. Is birth weight a risk factor for ischemic heart disease in later life? Am. J. Clin. Nutr., v. 85, n. 5, p. 1244-1250, May 2007.

INCERPI, E. K et al. Inhibition of endogenous glucocorticoid synthesis aggravates lung injury triggered by septic shock in rats. Int. J. Exp. Path., v. 96, n. 3, p. 133-139, Jun 2015.

JACKSON, A. A. et al. Increased systolic blood pressure in rats induced by a maternal low-protein diet is reversed by dietary supplementation with glycine. Clin. Sci. (Lond.), v. 103, n. 6, p. 633-639, Dec 2002.

JACOBI, J. Corticosteroid replacement in critically ill patients. Crit. Care Clin., v. 22, n. 2, p. 245-253, Apr 2006.

JAKUBOWSKI, A. et al. S-nitroso human serum albumin given after LPS challenge reduces acute lung injury and prolongs survival in a rat model of endotoxemia. Naunyn Schmiedebergs Arch. Pharmacol., v. 379, n. 3, p. 281-290, Mar 2009.

JAMES, W. P.; HAY, A. M. Albumin metabolism: effect of the nutritional state and the dietary protein intake. J. Clin. Invest., v. 47, n. 9, p. 1958-1972, Sep 1968. 
JANSSON, A. H.; ERIKSSON, C.; WANG, X. Effects of budesonide and Nacetylcysteine on acute lung hyperinflation, inflammation and injury in rats. Vascul. Pharmacol., v. 43, n. 2, p. 101-111, Aug 2005.

JANSSON, T.; POWELL, T. L. Role of the placenta in fetal programming: underlying mechanisms and potential interventional approaches. Clin. Sci. (Lond.), v. 113, n. 1, p. 1-13, Jul 2007.

JAQUET, D. et al. Insulin resistance early in adulthood in subjects born with intrauterine growth retardation. J. Clin. Endocrinol. Metab., v. 85, n. 4, p. 14011406, Apr 2000.

JOHN, C. D.; BUCKINGHAM, J. C. Cytokines: regulation of the hypothalamopituitary-adrenocortical axis. Curr. Opin. Pharmacol., v. 3, n. 1, p. 78-84, Feb 2003.

JOHNSON, E. R.; MATTHAY, M. A. Acute lung injury: epidemiology, pathogenesis, and treatment. J. Aerosol. Med. Pulm. Drug Deliv., v. 23, n. 4, p. 243-252, Aug 2010.

JORDAN, M. et al. Neutralization of endogenous IL-6 suppresses induction of IL-1 receptor antagonist. J. Immunol., v. 154, n. 8, p. 4081-4090, Apr 1995.

KAGAN, J. C. et al. TRAM couples endocytosis of Toll-like receptor 4 to the induction of interferon-beta. Nat. Immunol., v. 9, n. 4, p. 361-368, Apr 2008.

KAM, J. C. et al. Combination IL-2 and IL-4 reduces glucocorticoid receptor-binding affinity and $T$ cell response to glucocorticoids. J. Immunol., v. 151, n. 7, p. 34603466, Oct 1993.

KARROW, N. A. Activation of the hypothalamic-pituitary-adrenal axis and autonomic nervous system during inflammation and altered programming of the neuroendocrine-immune axis during fetal and neonatal development: lessons learned from the model inflammagen, lipopolysaccharide. Brain Behav. Immun., v. 20, n. 2, p. 144-158, Mar 2006.

KAWAI, T.; AKIRA, S. The role of pattern-recognition receptors in innate immunity: update on Toll-like receptors. Nat. Immunol., v. 11, n. 5, p. 373-384, May 2010.

KEMPNA, P.; FLUCK, C. E. Adrenal gland development and defects. Best Pract. Res. Clin. Endocrinol. Metab., v. 22, n. 1, p. 77-93, Feb 2008. 
KHORRAM, O. et al. Excess maternal glucocorticoids in response to in utero undernutrition inhibit offspring angiogenesis. Reprod. Sci., v. 21, n. 5, p. 601-611, May 2014.

KONRAD, F. M.; REUTERSHAN, J. CXCR2 in acute lung injury. Mediators Inflamm., v. 2012, p. 740987, 2012.

KOVACH, M. A.; STANDIFORD, T. J. Toll like receptors in diseases of the lung. Int . Immunopharmacol., v. 11, n. 10, p. 1399-1406, Oct 2011.

KUEHL, F. A., JR.; EGAN, R. W. Prostaglandins, arachidonic acid, and inflammation. Science, v. 210, n. 4473, p. 978-984, Nov 1980.

KUMAR, R.; THOMPSON, E. B. Gene regulation by the glucocorticoid receptor: structure:function relationship. J. Steroid Biochem. Mol. Biol., v. 94, n. 5, p. 383394, Apr 2005.

KYLE, U. G.; PICHARD, C. The Dutch Famine of 1944-1945: a pathophysiological model of long-term consequences of wasting disease. Curr. Opin. Clin. Nutr. Metab. Care, v. 9, n. 4, p. 388-394, Jul 2006.

LANDGRAF, M. A. Estudo da migração leucocitária em ratos desnutridos intrauterinamente. 2005. 146f. Tese (Doutorado em Farmacologia) - Instituto de Ciências Biomédicas, Universidade de São Paulo, São Paulo, 2005.

LANDGRAF, M. A. et al. Influence of age on the development of immunological lung response in intrauterine undernourishment. Nutrition, v. 24, n. 3, p. 262-269, Mar 2008.

LANDGRAF, M. A. et al. Intrauterine undernourishment alters TH1/TH2 cytokine balance and attenuates lung allergic inflammation in wistar rats. Cell. Physiol. Biochem., v. 30, n. 3, p. 552-562, 2012.

LANDGRAF, M. A. et al. Intrauterine undernutrition in rats interferes with leukocyte migration, decreasing adhesion molecule expression in leukocytes and endothelial cells. J. Nutr., v. 135, n. 6, p. 1480-1485, Jun 2005.

LANGLEY, S. C.; BROWNE, R. F.; JACKSON, A. A. Altered glucose tolerance in rats exposed to maternal low protein diets in utero. Comp. Biochem. Physiol. Physiol., v. 109, n. 2, p. 223-229, Oct 1994. 
LAWRENCE, T.; WILLOUGHBY, D. A.; GILROY, D. W. Anti-inflammatory lipid mediators and insights into the resolution of inflammation. Nat. Rev. Immunol. , v. 2, n. 10, p. 787-795, Oct 2002.

LE BEL, M.; BRUNET, A.; GOSSELIN, J. Leukotriene B4, an endogenous stimulator of the innate immune response against pathogens. J. Innate Immun., v. 6, n. 2, p. 159-168, 2014.

LE CONTEL, C. et al. Regulation of lipopolysaccharide-induced tumor necrosis factor production by cyclosporin A in mice primed with muramyl dipeptide. FEMS Immunol. Med. Microbiol., v. 11, n. 4, p. 297-305, Jul 1995.

LEECH, M. et al. Endogenous glucocorticoids modulate experimental antiglomerular basement membrane glomerulonephritis. Clin. Exp. Immunol., v. 119, n. 1, p. 161-168, Jan 2000.

LEONE, S. et al. Molecular structure of endotoxins from Gram-negative marine bacteria: an update. Mar. Drugs, v. 5, n. 3, p. 85-112, Sep 2007.

LESAGE, J. et al. Maternal undernutrition during late gestation induces fetal overexposure to glucocorticoids and intrauterine growth retardation, and disturbs the hypothalamo-pituitary adrenal axis in the newborn rat. Endocrinology, v. 142, n. 5, p. 1692-1702, May 2001.

LEY, K. et al. Getting to the site of inflammation: the leukocyte adhesion cascade updated. Nat. Rev. Immunol., v. 7, n. 9, p. 678-689, Sep 2007.

LIAO, J. et al. Role of corticosterone in TNF and IL-6 production in isolated perfused rat liver. Am. J. Physiol., v. 268, n. 3 Pt 2, p. R699-706, Mar 1995.

LILLYCROP, K. A. et al. Dietary protein restriction of pregnant rats induces and folic acid supplementation prevents epigenetic modification of hepatic gene expression in the offspring. J. Nutr., v. 135, n. 6, p. 1382-1386, Jun 2005.

LIM, H. Y. et al. Glucocorticoids exert opposing effects on macrophage function dependent on their concentration. Immunology, v. 122, n. 1, p. 47-53, Sep 2007.

LIN, H. Y.; MULLER, Y. A.; HAMMOND, G. L. Molecular and structural basis of steroid hormone binding and release from corticosteroid-binding globulin. Mol. Cell. Endocrinol., v. 316, n. 1, p. 3-12, Mar 2010. 
LISTON, C.; GAN, W. B. Glucocorticoids are critical regulators of dendritic spine development and plasticity in vivo. Proc. Natl. Acad. Sci. U. S. A., v. 108, n. 38, p. 16074-16079, Sep 2011.

LUCAS, A. et al. Nutrition in pregnant or lactating rats programs lipid metabolism in the offspring. Br. J. Nutr., v. 76, n. 4, p. 605-612, Oct 1996.

MALCHER-LOPES, R.; BUZZI, M. Glucocorticoid-regulated crosstalk between arachidonic acid and endocannabinoid biochemical pathways coordinates cognitive-, neuroimmune-, and energy homeostasis-related adaptations to stress. Vitam Horm, v. 81, p. 263-313, 2009.

MALKOSKI, S. P.; DORIN, R. I. Composite glucocorticoid regulation at a functionally defined negative glucocorticoid response element of the human corticotropinreleasing hormone gene. Mol. Endocrinol., v. 13, n. 10, p. 1629-1644, Oct 1999.

MARON-GUTIERREZ, T. et al. Insult-dependent effect of bone marrow cell therapy on inflammatory response in a murine model of extrapulmonary acute respiratory distress syndrome. Stem Cell Res. Ther., v. 4, n. 5, p. 123, Oct 2013.

MASCLANS, J. R. et al. Possible prognostic value of leukotriene $B(4)$ in acute respiratory distress syndrome. Respir. Care, v. 52, n. 12, p. 1695-1700, Dec 2007.

MATUTE-BELLO, G. et al. An official American Thoracic Society workshop report: features and measurements of experimental acute lung injury in animals. Am. J. Respir. Cell Mol. Biol., v. 44, n. 5, p. 725-738, May 2011.

MEDZHITOV, R. Origin and physiological roles of inflammation. Nature, v. 454, n. 7203, p. 428-435, Jul 2008.

MEDZHITOV, R. Inflammation 2010: new adventures of an old flame. Cell, v. 140, n. 6, p. 771-776, Mar 2010.

MEI, S. H. et al. Prevention of LPS-induced acute lung injury in mice by mesenchymal stem cells overexpressing angiopoietin 1. PLoS Med., v. 4, n. 9, p. e269, Sep 2007.

MELTZER, J. C. et al. Contribution of the adrenal glands and splenic nerve to LPSinduced splenic cytokine production in the rat. Brain Behav. Immun., v. 17, n. 6, p. 482-497, Dec 2003. 
MILLER, W. L.; AUCHUS, R. J. The molecular biology, biochemistry, and physiology of human steroidogenesis and its disorders. Endocr. Rev., v. 32, n. 1, p. 81-151, Feb 2011.

MILLS, K. H. TLR-dependent T cell activation in autoimmunity. Nat. Rev. Immunol., v. 11, n. 12, p. 807-822, Nov 2011.

MITCHELL, J. A. et al. Induction of cyclo-oxygenase-2 by cytokines in human pulmonary epithelial cells: regulation by dexamethasone. Br. J. Pharmacol., v. 113, n. 3, p. 1008-1014, Nov 1994.

MOORE, S. E. et al. Early immunological development and mortality from infectious disease in later life. Proc. Nutr. Soc., v. 65, n. 3, p. 311-318, Aug 2006.

MORROW, L. E. et al. Glucocorticoids alter fever and IL-6 responses to psychological stress and to lipopolysaccharide. Am. J. Physiol., v. 264, n. 5 Pt 2, p. R1010-1016, May 1993.

MULROW, P. J.; FRANCO-SAENZ, R. The adrenal renin-angiotensin system: a local hormonal regulator of aldosterone production. J. Hypertens., v. 14, n. 2, p. 173-176, Feb 1996.

NAKAMURA, K. Central circuitries for body temperature regulation and fever. Am. J. Physiol. Regul. Integr. Comp. Physiol., v. 301, n. 5, p. R1207-1228, Nov 2011.

NATHAN, C.; DING, A. Nonresolving inflammation. Cell, v. 140, n. 6, p. 871-882, Mar 2010.

NEEL, J. V. Diabetes mellitus: a "thrifty" genotype rendered detrimental by "progress"? Am. J. Hum. Genet., v. 14, p. 353-362, Dec 1962.

NELSON, P. J. et al. Genomic organization and transcriptional regulation of the RANTES chemokine gene. J. Immunol., v. 151, n. 5, p. 2601-2612, Sep 1993.

NORTHROP, J. P.; ULLMAN, K. S.; CRABTREE, G. R. Characterization of the nuclear and cytoplasmic components of the lymphoid-specific nuclear factor of activated T cells (NF-AT) complex. J. Biol. Chem., v. 268, n. 4, p. 2917-2923, Feb 1993.

OHLS, R. K. et al. Neutrophil pool sizes and granulocyte colony-stimulating factor production in human mid-trimester fetuses. Pediatr. Res., v. 37, n. 6, p. 806-811, Jun 1995. 
OPITZ, B. et al. Innate immune recognition in infectious and noninfectious diseases of the lung. Am. J. Respir. Crit. Care Med., v. 181, n. 12, p. 1294-1309, Jun 2010.

ORENTREICH, N. et al. Age changes and sex differences in serum dehydroepiandrosterone sulfate concentrations throughout adulthood. J. Clin. Endocrinol. Metab., v. 59, n. 3, p. 551-555, Sep 1984.

PARKER, J. C.; TOWNSLEY, M. I. Evaluation of lung injury in rats and mice. Am. J. Physiol. Lung Cell Mol. Physiol., v. 286, n. 2, p. L231-246, Feb 2004.

PEMBERTON, P. A. et al. Hormone binding globulins undergo serpin conformational change in inflammation. Nature, v. 336, n. 6196, p. 257-258, Nov 1988.

PEPE, P. E. et al. Clinical predictors of the adult respiratory distress syndrome. Am. J. Surg., v. 144, n. 1, p. 124-130, Jul 1982.

PEROGAMVROS, I.; RAY, D. W.; TRAINER, P. J. Regulation of cortisol bioavailability--effects on hormone measurement and action. Nat. Rev. Endocrinol., v. 8, n. 12, p. 717-727, Dec 2012.

PERRETTI, M.; AHLUWALIA, A. The microcirculation and inflammation: site of action for glucocorticoids. Microcirculation, v. 7, n. 3, p. 147-61, Jun 2000.

PERRETTI, M. et al. Serum corticosterone, interleukin-1 and tumour necrosis factor in rat experimental endotoxaemia: comparison between Lewis and Wistar strains. Br. J. Pharmacol., v. 110, n. 2, p. 868-874, Oct 1993.

PERUZZO, D. C. et al. Evidence that metyrapone in the presence of inflammation modulates cytokine mRNA expression. Cytokine, v. 52, n. 3, p. 184-189, Dec 2010.

PETRI, B.; PHILLIPSON, M.; KUBES, P. The physiology of leukocyte recruitment: an in vivo perspective. J. Immunol., v. 180, n. 10, p. 6439-6446, May 2008.

PETTIPHER, E. R. et al. Regulation of tumour necrosis factor production by adrenal hormones in vivo: insights into the antiinflammatory activity of rolipram. $\mathbf{B r}$. J. Pharmacol., v. 117, n. 7, p. 1530-1534, Apr 1996.

PRADER, A.; TANNER, J. M.; VON, H. G. Catch-up growth following illness or starvation. An example of developmental canalization in man. J. Pediatr., v. 62, p. 646-659, May 1963. 
PUGEAT, M. et al. Decreased immunoreactivity and binding activity of corticosteroidbinding globulin in serum in septic shock. Clin. Chem., v. 35, n. 8, p. 1675-9, Aug 1989.

RAE, M. T. et al. Antiinflammatory steroid action in human ovarian surface epithelial cells. J. Clin. Endocrinol. Metab., v. 89, n. 9, p. 4538-4544, Sep 2004.

RATHER, L. J. Disturbance of function (functio laesa): the legendary fifth cardinal sign of inflammation, added by Galen to the four cardinal signs of Celsus. Bull. N. Y. Acad. Med., v. 47, n. 3, p. 303-322, Mar 1971.

REHAN, V. K. et al. Metyrapone blocks maternal food restriction-induced changes in female rat offspring lung development. Reprod. Sci., v. 21, n. 4, p. 517-525, Apr 2014.

RHEN, T.; CIDLOWSKI, J. A. Antiinflammatory action of glucocorticoids--new mechanisms for old drugs. N. Engl. J. Med., v. 353, n. 16, p. 1711-1723, Oct 2005.

RIETSCHEL, E. T. et al. Bacterial endotoxin: molecular relationships of structure to activity and function. FASEB J., v. 8, n. 2, p. 217-225, Feb 1994.

ROGATSKY, I.; IVASHKIV, L. B. Glucocorticoid modulation of cytokine signaling. Tissue Antigens, v. 68, n. 1, p. 1-12, Jul 2006.

ROOZENDAAL, B.; BOHUS, B.; MCGAUGH, J. L. Dose-dependent suppression of adrenocortical activity with metyrapone: effects on emotion and memory. Psychoneuroendocrinology, v. 21, n. 8, p. 681-693, Nov 1996.

ROVAI, L. E.; HERSCHMAN, H. R.; SMITH, J. B. The murine neutrophilchemoattractant chemokines LIX, KC, and MIP-2 have distinct induction kinetics, tissue distributions, and tissue-specific sensitivities to glucocorticoid regulation in endotoxemia. J. Leukoc. Biol., v. 64, n. 4, p. 494-502, Oct 1998.

RYAN, G. B.; MAJNO, G. Acute inflammation. A review. Am. J. Pathol., v. 86, n. 1, p. 183-276, Jan 1977.

SAINIO, E. L.; LEHTOLA, T.; ROININEN, P. Radioimmunoassay of total and free corticosterone in rat plasma: measurement of the effect of different doses of corticosterone. Steroids, v. 51, n. 5-6, p. 609-622, May-Jun 1988. 
SAPER, C. B.; ROMANOVSKY, A. A.; SCAMMELL, T. E. Neural circuitry engaged by prostaglandins during the sickness syndrome. Nat. Neurosci., v. 15, n. 8, p. 10881095, Jul 2012.

SAPOLSKY, R. M. Why stress is bad for your brain. Science, v. 273, n. 5276, p. 749750, Aug 1996.

SCHACKE, H.; DOCKE, W. D.; ASADULLAH, K. Mechanisms involved in the side effects of glucocorticoids. Pharmacol. Ther., v. 96, n. 1, p. 23-43, Oct 2002.

SCHULZ, C. et al. A lineage of myeloid cells independent of Myb and hematopoietic stem cells. Science, v. 336, n. 6077, p. 86-90, Apr 2012.

SECKL, J. R. Prenatal glucocorticoids and long-term programming. Eur. J. Endocrinol., v. 151 Suppl 3, p. U49-62, Nov 2004.

SHER, E. R. et al. Steroid-resistant asthma. Cellular mechanisms contributing to inadequate response to glucocorticoid therapy. J. Clin. Invest., v. 93, n. 1, p. 33-39, Jan 1994.

SIITERI, P. K. et al. The serum transport of steroid hormones. Recent Prog. Horm. Res., v. 38, p. 457-510, 1982.

SIMPSON, S. A.; TAIT, J. F.; BUSH, I. E. Secretion of a salt-retaining hormone by the mammalian adrenal cortex. Lancet, v. 2, n. 6727, p. 226-228, Aug 1952.

$\mathrm{SOHI}, \mathrm{G}$. et al. Maternal protein restriction elevates cholesterol in adult rat offspring due to repressive changes in histone modifications at the cholesterol 7alphahydroxylase promoter. Mol. Endocrinol., v. 25, n. 5, p. 785-798, May 2011.

STRIZ, I. et al. Cytokine networking of innate immunity cells: a potential target of therapy. Clin. Sci. (Lond.), v. 126, n. 9, p. 593-612, May 2014.

SUGIMOTO, Y.; NARUMIYA, S. Prostaglandin E receptors. J. Biol. Chem., v. 282, n. 16, p. 11613-11617, Apr 2007.

TANIMURA, N. et al. Roles for LPS-dependent interaction and relocation of TLR4 and TRAM in TRIF-signaling. Biochem. Biophys. Res. Commun., v. 368, n. 1, p. 94-99, Mar 2008.

TANK, A. W.; LEE WONG, D. Peripheral and central effects of circulating catecholamines. Compr. Physiol., v. 5, n. 1, p. 1-15, Jan 2015. 
VAN AMERSFOORT, E. S.; VAN BERKEL, T. J.; KUIPER, J. Receptors, mediators, and mechanisms involved in bacterial sepsis and septic shock. Clin. Microbiol. Rev., v. 16, n. 3, p. 379-414, Jul 2003.

VAN FURTH, R. The mononuclear phagocyte system. Verh. Dtsch. Ges. Pathol., v. 64, p. 1-11, 1980.

VERHEGGEN, M. M. et al. Modulation of glucocorticoid receptor expression in human bronchial epithelial cell lines by IL-1 beta, TNF-alpha and LPS. Eur. Respir. J., v. 9, n. 10, p. 2036-2043, Oct 1996.

VIEAU, D. et al. HPA axis programming by maternal undernutrition in the male rat offspring. Psychoneuroendocrinology, v. 32 Suppl 1, p. S16-20, Aug 2007.

VISHWANATH, B. S. et al. Glucocorticoid deficiency increases phospholipase A2 activity in rats. J. Clin. Invest., v. 92, n. 4, p. 1974-1980, Oct 1993.

VO, T.; HARDY, D. B. Molecular mechanisms underlying the fetal programming of adult disease. J. Cell Commun. Signal., v. 6, n. 3, p. 139-153, Aug 2012.

VUGUIN, P. M. Animal models for small for gestational age and fetal programming of adult disease. Horm. Res., v. 68, n. 3, p. 113-123, 2007.

WANG, L. et al. Disruption of cytochrome P4501A2 in mice leads to increased susceptibility to hyperoxic lung injury. Free Radic. Biol. Med., v. 82, p. 147-159, May 2015.

WARE, L. B.; MATTHAY, M. A. The acute respiratory distress syndrome. N. Engl. J. Med., v. 342, n. 18, p. 1334-1349, May 2000.

WILKINSON, P. C. Surface and cell membrane activities of leukocyte chemotactic factors. Nature, v. 251, n. 5470, p. 58-60, Sep 1974.

WILKINSON, P. C.; MCKAY, I. C. Recognition in leucocyte chemotaxis. Studies with structurally modified proteins. Antibiot. Chemother. (1971), v. 19, p. 421-441, 1974.

WILLIAMS, T. J.; MORLEY, J. Prostaglandins as potentiators of increased vascular permeability in inflammation. Nature, v. 246, n. 5430, p. 215-217, Nov 1973. 
WU, D. X. et al. Lung lesions in experimental hydrostatic pulmonary edema: an electron microscopic and morphometric study. Exp. Lung Res., v. 21, n. 5, p. 711730, Sep-Oct 1995.

$\mathrm{XU}, \mathrm{Y}$. et al. Spred-2 deficiency exacerbates lipopolysaccharide-induced acute lung inflammation in mice. PLoS One, v. 9, n. 9, p. e108914, 2014.

YANG, R. et al. Suppression of NF-kappaB pathway by crocetin contributes to attenuation of lipopolysaccharide-induced acute lung injury in mice. Eur. J. Pharmacol., v. 674, n. 2-3, p. 391-396, Jan 2012.

ZHANG, T. Y.; DAYNES, R. A. Glucocorticoid conditioning of myeloid progenitors enhances TLR4 signaling via negative regulation of the phosphatidylinositol 3-kinaseAkt pathway. J. Immunol., v. 178, n. 4, p. 2517-2526, Feb 2007.

ZHANG, Y. C. et al. Glucocorticoid receptor expression on acute lung injury induced by endotoxin in rats. World J. Emerg. Med., v. 1, n. 1, p. 65-69, 2010. 